\title{
Revisión \\ Trichoderma: IMPORTANCIA AGRÍCOLA, BIOTECNOLÓGICA, Y SISTEMAS DE FERMENTACIÓN PARA PRODUCIR BIOMASA Y ENZIMAS DE INTERÉS INDUSTRIAL
}

\section{Review \\ Trichoderma: AGRICULTURAL AND BIOTECHNOLOGICAL IMPORTANCE, AND FERMENTATION SYSTEMS FOR PRODUCING BIOMASS AND ENZYMES OF INDUSTRIAL INTEREST}

\author{
Dulce Jazmín Hernández-Melchor ${ }^{1}$, Ronald Ferrera-Cerrato ${ }^{1}$, Alejandro Alarcón ${ }^{1 *}$ \\ ${ }^{1}$ Colegio de Postgraduados, Microbiología de Suelos, Posgrado de Edafología. Carretera México- \\ Texcoco km 36.5, Montecillo 56230, Estado de México, México. \\ * Autor de correspondencia: aalarconcp@gmail.com
}

\section{RESUMEN}

Trichoderma es un hongo cosmopolita cuya importancia radica en su capacidad de adaptación y producción de metabolitos, como enzimas, compuestos promotores de crecimiento vegetal, y compuestos volátiles, entre otros, de interés biotecnológico y ambiental. Este género es utilizado como agente de biocontrol contra hongos fitopatógenos debido a sus múltiples mecanismos de acción, destacando la antibiosis, el micoparasitismo, la competencia por espacio y nutrientes, y la producción de metabolitos secundarios. Varias especies de Trichoderma se han utilizado en sistemas acoplados de fermentación en sustratos sólidos o cultivos sumergidos, para degradar residuos lignocelulósicos y para generar energías alternativas como etanol. Los biorreactores como sistemas de fermentación optimizan las condiciones del cultivo para favorecer la generación de biomasa y metabolitos. Por lo anterior, el presente trabajo recopila información de los avances recientes del uso de Trichoderma en sistemas de producción y generación de subproductos de importancia para la agricultura y la biotecnología.

Palabras clave: biocontrol, bioprocesos, biorreactores, subproductos fúngicos

\section{ABSTRACT}

Trichoderma is a cosmopolitan fungus, which has the ability to adapt and produce metabolites such as enzymes, plant growth promoting compounds, and volatile compounds of biotechnological and environmental interest. This genus is used as a biocontrol agent against phytopathogenic fungi due to multiple mechanisms of action such as antibiosis, mycoparasitism, competition for space and nutrients, and the production of secondary metabolites. Likewise, several Trichoderma species have been used in coupled systems of fermentation in solid substrates or submerged cultures, to degrade lignocellulosic residues and to generate alternative energies such as ethanol. Bioreactors as fermentation systems optimize the cultural conditions to favor the generation of biomass and metabolites. Therefore, the present work reviews recent reports on the use of Trichoderma in systems for producing and generating important byproducts for agriculture and biotechnology.

Key words: biocontrol, bioprocesses, bioreactors, fungal byproducts

Recibido: 16 agosto 2018. Aceptado: 10 enero 2019. 


\section{INTRODUCCIÓN}

La agricultura ha proporcionado una fuente de alimento confiable para la población mundial; su importancia se ha reflejado en el movimiento intercultural de cultivos y razas ganaderas a lo largo de la historia, revolucionando las dietas y ayudando a reducir la pobreza (FAO, 2014). La seguridad alimentaria mundial es de gran importancia, por lo que un incremento de la inversión para investigación y desarrollo agrícola es necesario para aumentar la productividad de las granjas del mundo, especialmente en los países en desarrollo (Fuglie, 2016). A pesar de los esfuerzos en mejorar la economía agrícola y los sistemas de cultivo, se han presentado diversos problemas en la agricultura convencional y urbana, relacionados con el déficit de agua por la sobreexplotación de los mantos acuíferos, una creciente proliferación de plagas resistentes a insumos químicos, y la prohibición del uso de agroquímicos para su control (Dieleman, 2016).

América Latina, considerada una región rica en recursos renovables y no renovables, ha presentado problemas en los cultivos debido a sistemas agrícolas ineficientes, y a la limitada disponibilidad de nutrientes del suelo; por ello la aplicación de la biotecnología agrícola representa grandes beneficios para solucionar dichas problemáticas (Barragán-Ocaña y delValle-Rivera, 2016). El nitrógeno (N) es un claro ejemplo de nutriente con mayor déficit en los campos agrícolas, por lo que se han buscado estrategias para suministrarlo en los sistemas de cultivo, con uso de fertilizantes inorgánicos, estiércoles, y residuos de cosechas como abono o abonos verdes; sin embargo, el uso de fertilizantes químicos tiene ventajas y a la vez limitaciones, relacionadas con su costo y con la inducción de salinidad en el suelo por un uso excesivo (Komarek et al., 2017). Más aun, la agricultura moderna se ha vuelto dependiente del uso de plaguicidas químicos para controlar organismos fitopatógenos, lo cual ha provocado resistencia en las plagas, cambios en la diversidad microbiana edáfica, y contaminación ambiental (Chen et al., 2016).

El creciente daño ambiental generado por el uso de sustancias químicas para el control de enfermedades en las plantas, ha motivado el uso de alternativas biológicas, como es el caso de Trichoderma, el cual es un género fúngico de la rizosfera considerado simbionte oportunista de plantas, que es capaz de producir elicitores que inducen la defensa vegetal contra patógenos e insectos, ayudan a la solubilización de fósforo, y propician la síntesis de sustancias promotoras del crecimiento vegetal (Djonovic et al., 2007;
Hohmann et al., 2011). Trichoderma no solo se ha caracterizado por su uso como bioinoculante y como agente de biocontrol, sino también por su capacidad de generar enzimas capaces de degradar residuos orgánicos sólidos, por lo que puede coadyuvar en la mineralización y en la reutilización de los residuos (Diorio et al., 2003; Marques et al., 2017; Idris et al., 2017).

\section{Importancia agrícola del género Trichoderma como agente de biocontrol}

Las especies de Trichoderma predominan en ecosistemas terrestres (bosques o suelos agrícolas), tienen bajo requerimiento nutrimental pero relativamente amplio rango de temperatura $\left(25-30^{\circ} \mathrm{C}\right)$ para su crecimiento (Sandle, 2014). Además, poseen alta adaptabilidad a condiciones ecológicas y pueden crecer de manera saprofítica, interactúan con animales y plantas (Zeilinger et al., 2016), y se desarrollan en diversos sustratos, lo cual facilita su producción masiva para uso en la agricultura (Ramos et al., 2008). Es por ello que el estudio de la diversidad de especies de Trichoderma en diversos hábitats naturales, permite ampliar el conocimiento sobre su aporte biotecnológico, y su importancia ecológica y agrícola (Jaklitsch y Voglmayr, 2015; Torres-De la Cruz et al., 2015).

Diversas especies de este género están asociadas con la rizósfera de plantas o pueden relacionarse de manera endofítica, por lo que pueden promover el crecimiento y desarrollo de las plantas, mediante la producción de auxinas y giberelinas; también pueden producir ácidos orgánicos (glucónico, fumárico, y cítrico) que pueden disminuir el $\mathrm{pH}$ del suelo y propiciar la solubilización de fosfatos, magnesio, hierro y manganeso, los cuales son vitales para el metabolismo vegetal (Torres-De la Cruz et al., 2015; Sharma et al., 2017). Además, este género fúngico es importante para las plantas, al contribuir en el control de hongos fitopatógenos, ya que poseen propiedades micoparasítarias y antibióticas, por lo que algunas especies han sido catalogadas como excelentes agentes de control biológico de hongos causantes de enfermedades en diferentes plantas (Argumedo-Delira et al., 2009). Este hongo toma nutrientes de los hongos que parasita y de materiales orgánicos, ayudando a su descomposición, por lo cual las incorporaciones de materia orgánica y compostas favorecen su proliferación (Ramos et al., 2008).

Las propiedades antagónicas de Trichoderma hacia hongos patógenos se basan en la activación de múltiples mecanismos que incluyen la competencia por nutrientes y espacio, el micoparasitismo, la antibiosis, la promoción del crecimiento vegetal, e inducción de respuestas 
de defensa vegetal (de Aguiar et al., 2014; Sandle 2014; Vargas-Hoyos y Gilchrist-Ramelli, 2015). Durante el proceso de micoparasitismo, Trichoderma secreta enzimas que hidrolizan la pared celular de los hongos que parasita, siendo las más conocidas las proteasas, las quitinasas y las glucanasas (Marcello et al., 2010; GarcíaEspejo et al., 2016), y provocan la retracción de la membrana plasmática y la desorganización del citoplasma. También inhiben la germinación de esporas y la elongación del tubo germinativo (Romero-Cortes et al., 2016).

El efecto de diversas especies de Trichoderma sobre el biocontrol de enfermedades se ha estudiado en cultivos de importancia agronómica como tomate (Solanum lycopersicum), arroz (Oryza sativa), maíz (Zea mays), trigo (Triticum sp.), entre otros (Martínez et al., 2013; Diánez et al., 2016). Al-Hazmi y Javeed (2016) estudiaron cuatro concentraciones de inóculo de T. harzianum y $T$. viride para el control de Meloidogyne javanica en tomate, encontrando que las concentraciones estudiadas suprimen la reproducción del nematodo e incrementan el crecimiento de la planta. Además, cabe destacar que no solo es importante el agente de biocontrol utilizado, sino también la forma de aplicación del mismo. Por ejemplo, de Aguiar et al. (2014) estudiaron el antagonismo de Trichoderma spp. contra Sclerotinia sclerotiorum en cultivos de tomate hibrido Heinz 7155, destacando la aplicación del biofungicida mediante irrigación por goteo, favoreciendo el control de la enfermedad y el aumento en el rendimiento de la fruta. Charoenrak y Chamswarng (2016) estudiaron el uso de T. asperellum 01-52 y CB-Pin-01 en cultivo fresco y en forma de pellets, como agente de biocontrol del agente causal de la enfermedad de la decoloración de semillas de arroz (O. sativa) en Tailandia, encontrando que ambas formulaciones incrementaron la altura de la planta, el número de tallos, el peso de las semillas, y el rendimiento total de arroz, en comparación con el testigo no tratado; además de disminuir la incidencia de la enfermedad en las plantas.

\section{Mineralización/biodegradación de sustratos orgánicos por Trichoderma}

Trichoderma es un hongo metabólicamente versátil, capaz de utilizar un amplio rango de biomasa vegetal, incluyendo oligosacáridos como sucrosa, rafinosa y polisacáridos como celulosa, inulina, quitina, pectina, y almidón (Sandle, 2014); así como sustratos más complejos como suero de leche, hidrocarburos del petróleo e incluso plaguicidas, contribuyendo potencialmente a su degradación (Guoweia et al., 2011). También es capaz de utilizar y degradar residuos lignocelulósicos que están constituidos de celulosa (40-55\%), hemicelulosa (25-50\%), y lignina (10-40\%), dependiendo si la fuente es madera dura, madera blanda, o rastrojos (la Grange et al., 2010).

En el proceso de fermentación de residuos lignocelulósicos se logra obtener productos hidrolíticos como hexosas monoméricas (glucosa, manosay galactosa), y pentosas (xilosay arabinosa) (Dashbatan et al., 2009). Los organismos capaces de degradar celulosa secretan un complejo de celulasas con diferentes especificidades y modos de acción. Este complejo incluye endoglucanasas (endo-1,4- $\quad \beta$-glucanasas), celobiohidrolasas o exoglucanasas y $\beta$-glucosidasas (Castrillo et al., 2015). La lignina es degradada por la interacción de diversas enzimas extracelulares, principalmente fenoloxidasas (lacasas) y peroxidasas (manganeso-peroxidasa y ligninaperoxidasa) (Barreto et al., 2011) produciendo compuestos fenólicos tales como ácido ferulico, ácido vanilico, entre otros, y alcoholes como guaiacol y catecol (Ibraheem y Ndimba, 2013). El xilano es el componente mayoritario de la hemicelulosa, la $\beta$-1,4-endoxilanasa y la $\beta$ -xilosidasa son las enzimas responsables de la hidrólisis de la cadena principal y representan los principales componentes del sistema xilanolítico (Dashtban et al., 2009). La conversión biológica de biomasa lignocelulósica a azúcares simples está constituida de dos pasos básicos, que constan de un pretratamiento físico o químico de los residuos, y de la hidrolisis enzimática de los mismos (Fig. 1).

\section{Metabolitos secundarios: enzimas y otros subproductos derivados de Trichoderma}

Este hongo tiene relevancia industrial y ecológica ya que puede sintetizar y liberar enzimas como celulasas; además de producir diversos metabolitos secundarios como auxinas y giberelinas (Zeilinger et al., 2016). Trichoderma ha sido considerado como una fuente práctica de enzimas incluyendo aquellas utilizadas en el área alimenticia, tales como celulasas, glucanasas, xilanasas, pectinasas, y laminarinasas (Sandle, 2014).

En la Tabla 1 se presentan ejemplos de enzimas producidas por diversas especies de Trichoderma. Así, T. reesei es una de las especies más utilizadas a nivel industrial para la producción de enzimas por su capacidad de degradar celulosa, aunque el costo del medio de cultivo es la limitante primordial en estos procesos, por lo que para la producción de estas enzimas se han utilizado otros sustratos, que aportan la misma fuente de carbono, como lo son los residuos lignocelulósicos tales como paja de trigo, bagazo, lodos de papel 

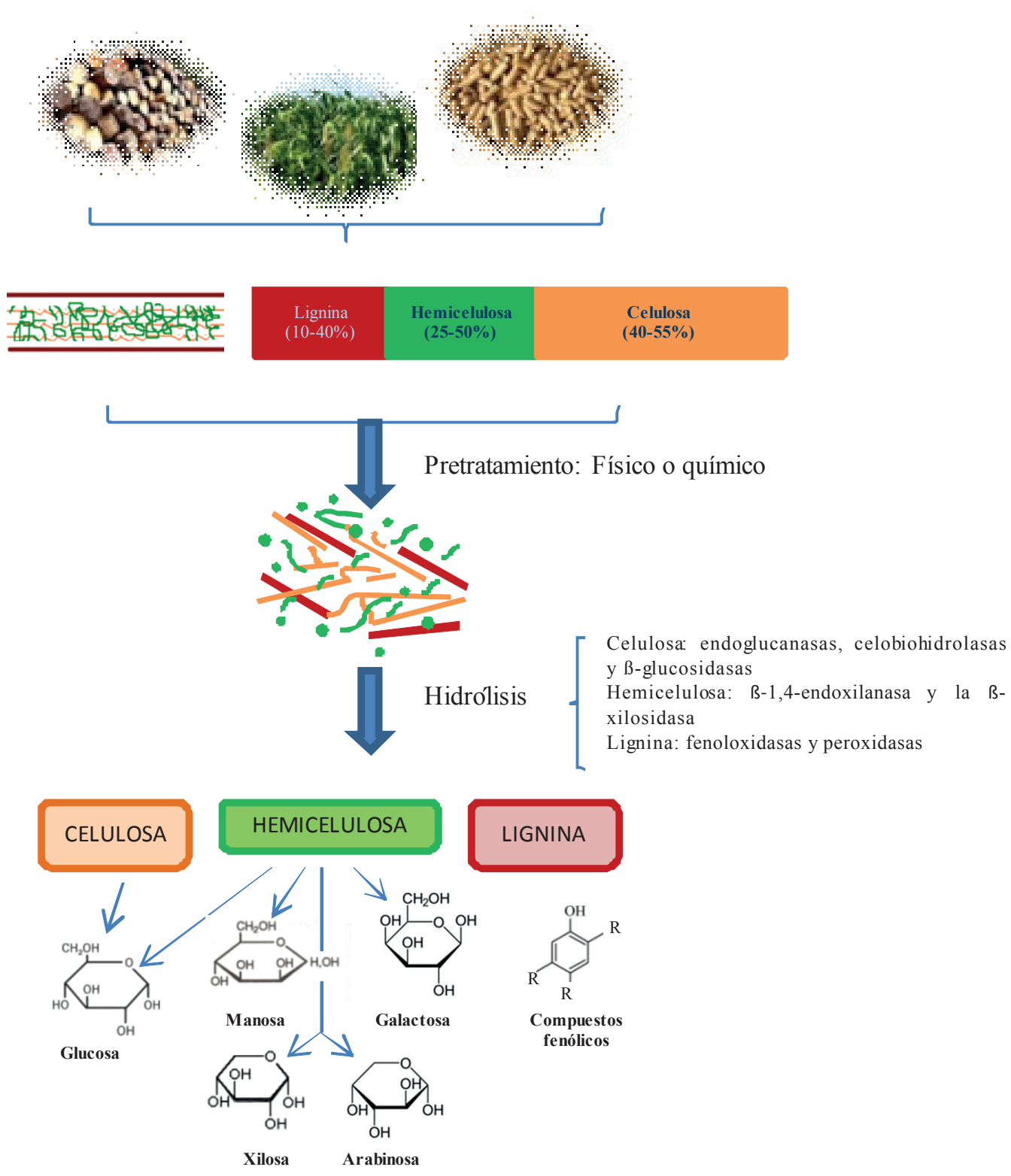

Fig. 1. Productos de la hidrólisis enzimática de residuos lignocelulósicos.

Fig. 1. Products of enzymatic hydrolysis of lignocellulosic residues.

usado, residuos de papel de periódico, y madera de álamo (Guoweia et al., 2011).

Cada año, la industria textil genera grandes cantidades de efluentes producidos durante la manufactura de colorantes, provocando severos problemas ambientales por su mala disposición, por lo que el tratamiento biológico es el método menos costoso y amigable con el ambiente para tratar de resolver este problema. Por ejemplo, las enzimas lacasas se han utilizado para decolorar colorantes sintéticos, sin generar intermediarios tóxicos. Trichoderma atroviride, T. harzianum, y T. longibrachiatum pueden ser fuentes de producción de estas enzimas al degradar sustratos lignocelulósicos; por lo que diversos autores han llevado a cabo su inmovilización para utilizarlas en el tratamiento de dichos efluentes (Bagewadi et al., 2017).

Otros metabolitos secundarios de importancia agrícola sintetizados por especies de Trichoderma se relacionan con compuestos volátiles y no volátiles con actividad antimicrobiana y defensa vegetal, destacando diterpenos tetracíclicos (por ejemplo, harziandione), sesquiterpenos (por 
Tabla 1. Ejemplos de enzimas producidas por Trichoderma empleando diferentes sustratos Table 1. Examples of enzymes produced by Trichoderma using different substrates

\begin{tabular}{|c|c|c|c|}
\hline Cepa de Trichoderma & Enzima & Sustrato & Referencia \\
\hline T. harzianum & Quitinasas & Medio mineral & Azin et al., 2007 \\
\hline T. viride ATCC & Celulasas & $\begin{array}{l}\text { Salvado de trigo, medio líquido } \\
\text { Mandel's }\end{array}$ & $\begin{array}{l}\text { Subramaniyam y } \\
\text { Vimala, } 2012\end{array}$ \\
\hline T. reesei & $\begin{array}{l}\text { Celulasas, } \\
\text { Hemicelulasas }\end{array}$ & Medio mineral & Sandle, 2014 \\
\hline T. longibratum & Xilanasas & Medio mineral & Sandle, 2014 \\
\hline T. asperellum & $\begin{array}{l}\text { Glucanasas, } \\
\text { quitinasas }\end{array}$ & $\begin{array}{l}\text { Almidon, pared celular de } \\
\text { Rhizoctonia (PCRS), quitina } \\
\text { coloidal, laminarina }\end{array}$ & $\begin{array}{l}\text { Vargas-Hoyos y } \\
\text { Gilchrist-Ramelli, } 2015\end{array}$ \\
\hline T. koningiopsis & $\begin{array}{l}\text { endo-1,4- } \beta \text { - } \\
\text { glucanases }\end{array}$ & Aserrín de pino & Castrillo et al., 2015 \\
\hline $\begin{array}{l}\text { T. longibrachiatun } / \mathrm{H} \text {. } \\
\text { sagamiensis }\end{array}$ & $\begin{array}{l}\text { celulasas, } \\
\text { glucanasas e } \\
\text { hidrolasas }\end{array}$ & & $\begin{array}{l}\text { Torres-De la Cruz et al., } \\
2015\end{array}$ \\
\hline T. inhamatum BOL 12 QD & $\begin{array}{l}\text { Celulasas } \\
\text { Quitinasas } \\
\text { proteasas }\end{array}$ & $\begin{array}{l}\text { Quitina coloidal } \\
\text { carboximetilcelulosa }\end{array}$ & Garcia-Espejo et al., 2016 \\
\hline T. viride $\mathrm{EF}-8$ & Pectinasas & $\begin{array}{l}\text { Pieles de cebolla Egipcia } \\
\text { (Allium cepa L.) }\end{array}$ & $\begin{array}{l}\text { Abdel-Mohsen et al., } \\
2016\end{array}$ \\
\hline T. viride IR05 & Xilanasa & $\begin{array}{l}\text { Salvado de trigo, arroz pulido, } \\
\text { cáscara de arroz, harina de soja, } \\
\text { harina de girasol, bagazo de } \\
\text { caña de azúcar }\end{array}$ & Soccol et al., 2017 \\
\hline T. asperellum & Hemicelulasas & $\begin{array}{l}\text { Manojo de palma de aceite y } \\
\text { frutos secos }\end{array}$ & Ajijolakewu et al., 2017 \\
\hline
\end{tabular}

ejemplo, tricotecenos, tricodermin, y harzianum A), y el triterpeno viridin (Zeilinger et al., 2016), que confieren sus propiedades como agente de biocontrol hacia organismos fitopatógenos (Asad et al., 2015; Chen et al., 2016). El éxito de Trichoderma como biocontrolador se basa en su capacidad de sintetizar compuestos antagónicos (proteínas, enzimas y antibióticos), y sustancias promotoras de crecimiento (vitaminas y hormonas), beneficiando así a los cultivos agrícolas (Navaneetha et al., 2015).

Importancia biotecnológica del género Trichoderma: Aplicaciones industriales

Trichoderma está entre los agentes de control biológico más exitosos en la agricultura, formando parte de más del $60 \%$ de los biofungicidas registrados en el mundo. Este microorganismo está presente en el mercado como bioplaguicida, biofertilizante, promotor del rendimiento $\mathrm{y}$ crecimiento vegetal, y como solubilizador de nutrientes en campos agrícolas o descomponedor de materia orgánica (Vinale et al., 2008; Charoenrak y Chamswarng, 2016). Trichoderma se produce en formulaciones sólidas y líquidas con inóculo viable (hifas, clamidosporas y conidias) para usarlo como agente de biocontrol (Cumagun, 2014). Ambas formulaciones requieren el secado para obtener un producto estable con prolongada vida de anaquel; así, el secado por aspersión es el más utilizado a escala industrial por su bajo costo y eficiencia (Morgan et al., 2006). La mayoría de estos bioformulados se preparan a base de las especies T. viride, T. virens, y en mayor proporción T. harzianum (Vargas-Hoyos y Gilchrist-Ramelli, 2015).

Para asegurar el éxito de Trichoderma como biocontrolador, éste debe ser inmovilizado en ciertos portadores, y preparado en formulaciones de fácil aplicación, almacenamiento, comercialización y aplicación en campo. Algunas formulaciones comerciales de este microorganismo corresponden a talcos, vermiculita-salvado de trigo, gránulos de pesta (masa cohesiva hecha de harina de trigo, rellenos y otros aditivos que contienen al hongo), cáscara de café, aceite vegetal o mineral, y desechos de banano (Musa paradisiaca) (Kumar et al., 2014). 
También se puede encontrar disponible en polvo, Pyrex-biomasa, pellets de alginato, entre otras; no obstante, existen diferentes problemas asociados con el tipo de formulación seleccionada para cada caso de aplicación; por ejemplo, las formulaciones en polvo provocan la desecación de conidios, y por tanto, la pérdida de viabilidad de propágulos a los tres o seis meses de almacenamiento, afectando su eficiencia y comercialización. Como alternativa, los aerosoles foliares favorecen la supervivencia del agente fúngico durante periodos más largos (Navaneetha et al., 2015).

India es el mayor distribuidor de estos productos, destacando el uso de especies certificadas de $T$. asperellum, $T$. atroviride, $T$. gamsii, T. hamatum, T. harzianum, T. polysporum, T. virens, y T. viride; otros países que se destacan por la producción de este microorganismo son Bélgica, Chipre, Francia, Italia, España, Suecia, Eslovenia, Reino Unido, Turquía, Estados Unidos de América, Canadá, Sudáfrica, Marruecos, Vietnam, Australia, y Nueva Zelanda. De las formulaciones comerciales, el $67 \%$ de ellas están constituidas por una sola especie de Trichoderma, el resto incluye combinaciones de dos o más especies del mismo género, o su mezcla con especies de hongos formadores de micorrizas, bacterias $\mathrm{u}$ otros compuestos biológicos (Woo et al., 2014). En la Tabla 2 se presentan las formulaciones comerciales de Trichoderma que se utilizan en cada región del mundo.

La producción comercial de bioplaguicidas en México se lleva a cabo en aproximadamente 68 plantas industriales, en las que se reproducen 37 agentes de control biológico. De estos agentes, 14 son especies de Trichoderma (T. fasciculatum, $T$. harzianum, y T.viride) utilizadas como ingredientes activos en la formulación de los bioplaguicidas, a los que se adiciona un ingrediente inerte, una sustancia o coadyuvante que facilita su manejo, aplicación y efectividad (García de León y Mier, 2010). En la Tabla 3 se muestran ejemplos de empresas mexicanas que producen especies de Trichoderma para el control biológico.

Otro uso industrial de este hongo es la producción de enzimas, donde T. viride y T. reesei producen abundantes enzimas hidrolíticas como celulasas, que son utilizadas en la industria de alimentos, piensos, textiles, detergentes para ropa, pulpa y papel, fermentación de alcohol de grano, procesamiento de almidón, en la industria farmacéutica y cervecera, y para la producción de biocombustibles (Ahamed y Vermette, 2008a; Castrillo et al., 2015). La especie más estudiada y producida para estos propósitos es T. reesei, que tiene la capacidad de producir enzimas a bajo costo por la conversión de biomasa vegetal, obteniendo además bioproductos de interés industrial como azúcares y bioetanol (Callow et al., 2016). Además de celulasas, la producción de lacasas por especies de Trichoderma tiene importancia biotecnológica para la industria de la pulpa y el papel, así como para el bio-blanqueo, la síntesis orgánica (catalizan la oxidación de sustratos fenólicos), la clarificación de jugo y vino, la biorremediación de suelos contaminados con insecticidas, y como herramientas de diagnóstico médico (electroinmunoensayos con biosensores o detección de neurotransmisores), y además para la decolorización de colorantes tóxicos generados por la industria textil (Bagewadi et al., 2017).

\section{Sistemas de producción (biorreactores) de Trichoderma: Fermentación en estado sólido y fermentación sumergida}

La fermentación es el proceso en el cual los sustratos sólidos o líquidos son convertidos por microorganismos procariontes (bacterias) y eucariontes (microalgas, levaduras, hongos) en productos de alto valor agregado, útiles para el ser humano (Vuppala et al., 2015; Ashok et al., 2017). De manera general, la fermentación puede ser en estado sólido o en fermentación sumergida (líquida), para la producción de compuestos bioactivos a nivel industrial (Subramaniyamy Vimala, 2012).

La fermentación sólida es aplicada para procesos en los cuales materiales insolubles en agua son utilizados para el crecimiento del microorganismo (la cantidad de agua no debe exceder la capacidad de saturación del sólido en el cual crecerá el microorganismo). Los sustratos que pueden utilizarse son salvado de trigo, bagazo de caña (subproducto de la fabricación de azúcar) y pulpa de papel (Subramaniyam y Vimala, 2012; Kumar et al., 2014; Vuppala et al., 2015).

La fermentación líquida (sumergida) es aplicada en procesos en los cuales los materiales solubles en agua son utilizados para el crecimiento de los microorganismos y para la multiplicación de biomasa, por lo que el medio seleccionado debe ser barato, de fácil adquisición y con apropiado balance nutrimental, siendo los más utilizados melazas y caldos de cultivo que contienen sales minerales, vitaminas, azúcares, etc.

La fermentación sólida y la fermentación líquida (sumergida) han sido utilizadas para producir Trichoderma y sus compuestos bioactivos tales como enzimas (Kumar et al., 2014). En la Tabla 4 se presentan algunas ventajas y desventajas de cada sistema de fermentación.

\section{a) Fermentación en estado sólido}

La producción de Trichoderma en fermentación en estado sólido a pequeña escala, es poco 
Tabla 2. Cantidad de formulaciones comerciales de Trichoderma registradas en diferentes regiones del mundo.

Table 2. Quantity of commercial formulations of Trichoderma registered in different regions of the world.

\begin{tabular}{lccc}
\hline Región geográfica & $\begin{array}{c}\text { Número de formulaciones } \\
\text { comerciales }\end{array}$ & Especie sola & Mezcla de especies \\
\hline África & 9 & 7 & 2 \\
Asia & 100 & 77 & 23 \\
Europa & 42 & 24 & 18 \\
Norte América & 29 & 16 & 13 \\
Pacifico & 24 & 19 & 5 \\
Sur-Centro de América & 40 & 19 & 21 \\
\hline
\end{tabular}

Tomado de Woo et al., 2014.

Tabla 3. Empresas productoras de formulaciones de Trichoderma en México. Table 3. Companies that produce Trichoderma formulations in Mexico.

\begin{tabular}{cl}
\hline \multicolumn{1}{c}{ Cepa } & \multicolumn{1}{c}{ Planta productora } \\
\hline Trichoderma fasciculatum & Laboratorio Nafex, Edo. de Méx. \\
Trichoderma harzianum & Leticia de Paz Hernández, Edo. de Méx. \\
& Agricultura Nacional S.A. de C.V., Puebla \\
& Agrobiológicos del Noroeste S.A. de C.V., Sinaloa \\
& Agrobiosol de México S.A. de C.V., Sinaloa \\
& Alta Tecnológica Agrotécnica S.P.R. de R.L. de C.V., Jalisco \\
& Bio-Bich Uruapan, Michoacan \\
& Control Biológico A.C., Chihuahua \\
& Desarrollo Lácteo S.P.R. de R.L., Durango. \\
& Laboratorio Nafex, Edo. de Méx. \\
& Laboratorios y Servicios Agrobiológicos S.A. de C.V., Sinaloa \\
& Leticia de Paz Hernández, Edo. de Méx. \\
& Naturalmente Puresa S.A. de C.V., Durango \\
& Nocon S.A. de C.V., Edo. de Méx. \\
& Organismos Benéficos de Nayarit, Nayarit \\
& Agro Fisher S.P.R. de R.L., Michoacán \\
& Agroindustria Fungi-Agrícola de Oriente S.P.R. de R.I., Tepeaca, \\
& Puebla \\
& Ultraquimia Agrícola S.A. de C.V., CDMX \\
Islavel S.A. de C.V., Puebla. \\
Leticia de Paz Hernández, Edo. de Méx. \\
Nocon S.A. de C.V., Edo. de Méx.
\end{tabular}

Tomado de García de León y Mier, 2010.

costosa pero voluminosa, ya que requiere amplio espacio para su producción, inoculación y almacenamiento, incluyendo el secado y la molienda (Cumagun, 2014). A través de los años se han diseñado diversas configuraciones para resolver estos inconvenientes, entre los más estudiados encontramos: bandejas, cuartos incubados, columnas de lecho empacado, lecho múltiple, biorreactores de tipo tambor rotatorio múltiple, etc. (Ruiz et al., 2012; Ashok et al., 2017). Se destaca el uso de múltiples sustratos sólidos complejosconsiderados residuosagroindustriales, como bagazo de caña, madera, hojas, salvado de trigo, pulpa de café, cáscara de frutas, entre otros, para generar productos de alto valor agregado como enzimas, aromas, promotores 
Tabla 4. Ventajas y desventajas de la fermentación en estado sólido y fermentación sumergida. Table 4. Advantages and disadvantages of fermentations in solid and submerged state.

\begin{tabular}{|c|c|c|c|}
\hline $\begin{array}{l}\text { Tipo de } \\
\text { fermentación }\end{array}$ & Ventajas & Desventajas & Referencias \\
\hline $\begin{array}{l}\text { Fermentación } \\
\text { en estado sólido }\end{array}$ & $\begin{array}{l}\text { Bajo capital y costos de operación } \\
\text { Facilidad de manejo de grandes } \\
\text { volúmenes de residuos como } \\
\text { sustrato } \\
\text { Reutilización de residuos } \\
\text { producto de la fermentación } \\
\text { Menos consumo de energía } \\
\text { Menos efluentes de aguas } \\
\text { residuales } \\
\text { Menor número de operaciones } \\
\text { unitarias de separación y } \\
\text { purificación de metabolitos } \\
\text { Menos susceptible a inhibición } \\
\text { por sustrato }\end{array}$ & $\begin{array}{l}\text { Tiempo de fermentación } \\
\text { largo } \\
\text { No es posible manejar } \\
\text { un sistema de aireación } \\
\text { eficiente } \\
\text { Sistema heterogéneo } \\
\text { Difícil escalamiento a } \\
\text { nivel industrial } \\
\text { Poco control de la } \\
\text { temperatura } \\
\text { Cambios críticos en } \\
\text { la actividad del agua } \\
\text { durante el proceso } \\
\text { Difícil mezclado } \\
\text { Difícil esterilización } \\
\text { y control de } \\
\text { contaminantes } \\
\text { Se presenta gradientes } \\
\text { de concentración de } \\
\text { nutrientes a través del } \\
\text { tiempo } \\
\text { Es necesaria la adición } \\
\text { de macro y micro } \\
\text { nutrientes }\end{array}$ & $\begin{array}{l}\text { Diorio et al., } 2003 \\
\text { Subramaniyamy } \\
\text { Vimala, } 2012 \\
\text { Ashok et al., } 2017 \\
\text { Canabarro et al., } \\
2017 \\
\text { Soccol et al., } 2017\end{array}$ \\
\hline $\begin{array}{l}\text { Fermentación } \\
\text { sumergida }\end{array}$ & $\begin{array}{l}\text { Sistema homogéneo } \\
\text { Fácil mezclado } \\
\text { Sistemas automatizados de } \\
\text { instrumentación y control para el } \\
\text { monitoreo de temperatura, pH, } \\
\text { oxígeno disuelto, aireación, etc. } \\
\text { Fácil esterilización y control de } \\
\text { contaminantes } \\
\text { Los nutrientes son } \\
\text { uniformemente distribuidos a } \\
\text { través de la fermentación } \\
\text { Cantidad de inóculo baja } \\
\text { Favorece la formación de pellets } \\
\text { por lo que facilita su separación } \\
\text { después de la fermentación } \\
\text { Factible su escalamiento }\end{array}$ & $\begin{array}{l}\text { Generación de grandes } \\
\text { cantidades de efluentes } \\
\text { Costoso por la } \\
\text { implementación de los } \\
\text { sistemas de monitoreo y } \\
\text { control }\end{array}$ & $\begin{array}{l}\text { Subramaniyamy } \\
\text { Vimala, } 2012 \\
\text { Ashok et al., } 2017 \\
\text { Soccol et al., } 2017\end{array}$ \\
\hline
\end{tabular}


de crecimiento, biocombustibles, etc. (Fadel et al., 2015; Soccol et al., 2017). La fermentación en estado sólido ha sido ampliamente utilizada en procesos de biorremediación y biodestoxificación de compuestos peligrosos, y en la producción de enzimas terapéuticas, y metabolitos secundarios (Ashok et al., 2017).

Para analizar la acumulación de productos durante fermentación en estado sólido es necesario conocer las sustancias que se forman durante el crecimiento y el desarrollo del organismo, a fin de comprender su biosíntesis. Una estrategia propuesta para describir lo anterior, es el modelamiento matemático de la cinética de cultivo fúngico que permita describir y escalar su proceso productivo (Barreto et al., 2011; Ashok et al., 2017).

Además, se han diseñado sistemas acoplados para la generación de bioproductos mediante fermentación en estado sólido, como la sacarificación-fermentación para la producción de etanol utilizando residuos lignocelulósicos. Otro ejemplo, basado en un sistema acoplado de fermentación para optimizar la producción de celulasas utilizando fermentadores de bandeja de $50 \mathrm{~g}$ de capacidad en una cámara controlada llamada "koji rooms" utilizando T. reesei, han logrado la hidrólisis enzimática de sorgo, convirtiendo los azúcares en etanol con $80 \%$ de eficiencia, con ayuda de Saccharomyces cerevisiae; además, este sistema permitió incrementar hasta 3 veces la productividad enzimática en comparación con el método tradicional, utilizando el mismo sustrato (Idris et al., 2017).

\section{b) Fermentación sumergida (líquida)}

La fermentación sumergida se lleva a cabo en tanques especializados (biorreactores) considerando condiciones anaerobias o bien aerobias, en las que el suministro de aire puede realizarse mediante un sistema de agitación neumática o mecánica, o su combinación (Vuppala et al., 2015). Las configuraciones de biorreactores aerobios más estudiadas a diferentes escalas de producción, contemplan tanques agitados, columna de burbuja, y air-lift (de Jesús et al., 2017); estos sistemas de producción son ampliamente utilizados para la producción de enzimas a nivel industrial (Ashok et al., 2017). Así, más del 75\% de las enzimas microbianas son producidas por fermentación sumergida en comparación con la fermentación en estado sólido (Subramaniyam y Vimala, 2012).

Trichoderma es un microorganismo anaerobio facultativo que requiere para su crecimiento, bajas concentraciones de oxígeno en el sistema de fermentación (Ramos et al., 2008). La especie $T$. reesei ha sido ampliamente estudiada en biorreactores tipo tanque agitado para producir celulasas a nivel industrial o para convertir la biomasa lignocelulósica en etanol, basándose en un proceso simultáneo de fermentaciónsacarificación (Ahamed y Vermette, 2008a). Para incrementar la producción de celulasas en biorreactores tipo tanque agitado se han aplicado estrategias como la implementación de medios de cultivo, co-cultivos de hongos, operación en lotes alimentados y adicionados con inductores (lactosa o ácido lactobiónico), entre otras (Ahamed y Vermette, 2008a; b).

Ahamed y Vermette (2008b) trabajaron con un cultivo mixto de Trichoderma reesei y Aspergillus niger en un biorreactor tipo tanque agitado en un medio que contenía celulosa y extracto de levadura como elementos principales, reportando un incremento en las actividades enzimáticas y porcentaje de celulosa utilizada $(89,4 \%)$ respecto a la producción con monocultivos de las cepas puras. Dichos autores a través de diferentes estudios en biorreactores, lograron concluir que los requerimientos básicos que deben cubrirse para favorecer un rendimiento elevado de enzimas hidrolíticas utilizando sustratos complejos como la celulosa, se relacionan con una fuente de carbono complementaria, una fuente de nitrógeno, además de sulfatos, fosfatos y cloruros, y con el uso de co-cultivos fúngicos para mejorar los procesos (Ahamed y Vermette, 2008a; Ahamed y Vermette, 2009). Con la finalidad de optimizar estos procesos, se han utilizado herramientas estadísticas que ayudan a estudiar la correlación de los componentes del medio de cultivo y la producción de enzimas de interés industrial utilizando sustratos complejos en biorreactor (Delabona et al., 2013).

Los biorreactores tipo tanque agitado son los más utilizados a escala piloto e industrial para producir biomasa y enzimas a partir de $T$. reesei, por lo que el conocimiento y la predicción del mezclado y la transferencia de masa en biorreactores agitados son fundamentales para desarrollar bioprocesos biotecnológicos y su escalamiento. Bach et al. (2017) caracterizaron el tiempo de mezclado y el coeficiente volumétrico de transferencia de masa de un biorreactor de $350 \mathrm{~L}$ para predecir y mejorar la producción de biomasa fúngica mediante dinámica de fluidos computacional (CFD). Sin embargo, este tipo de sistemas presentan diversos inconvenientes, ya que el estrés hidrodinámico excesivo, generado por la agitación mecánica, provoca la ruptura del micelio en forma de pellets y el daño de las hifas, disminuyendo así su rendimiento. Para mitigar este inconveniente se ha propuesto el uso de biorreactores air-lift (sistema de agitación neumática que pone en contacto las fases 
gas-líquido a través de un bucle interno que genera dos zonas (ascendente y descendente), permitiendo así el mezclado y la transferencia de masa (Ahamed y Vermette, 2010; Callow et al., 2016).

Un sistema novedoso de biorreacción para la generación de metabolitos de interés industrial mediante el uso de enzimas producidas por Trichoderma, son los biorreactores de membrana, los cuales consisten en un sistema de separación de membrana acoplado a un fermentador, que permite la reutilización continua de enzimas. Esta característica es crucial para reducir costos del proceso, y asegurar mayor capacidad y rendimiento, funcionando a densidades celulares elevadas y evitando la inhibición del producto. Este sistema de biorreacción se ha utilizado para generar ácido láctico, que es precursor del ácido poliláctico (polímero biodegradable de relevancia industrial), a través de una fermentación simultánea, utilizando material lignocelulósico como sustrato, y enzimas celulolíticas y xilanolíticas para la sacarificación (Marques et al., 2017).

\section{Diseño de bioprocesos para la producción de biomasa y metabolitos de Trichoderma}

El bioproceso es definido como un proceso que utiliza células de microorganismos en cultivo o enzimas para manufacturar productos o para completar una transformación química (Kent et al., 2017). En general, las operaciones que comprenden los bioprocesos a escala comercial se han dividido en operaciones previas ("upstream") y en operaciones posteriores ("downstream"). Dentro de las primeras se considera la preparación del medio, la esterilización y el funcionamiento del biorreactor, y en las segundas, se involucran todas aquellas operaciones de bioseparación que ayudan a la recuperación y purificación del producto proveniente del biorreactor. Estas últimas pueden representar hasta $60 \%$ del costo total de producción (Doran, 1998; Tejeda, 2011). El bioproceso de producción a gran escala de hongos filamentosos es de gran importancia debido a su amplio rango de productos de valor agregado que pueden generar; sin embargo, es necesario optimizar algunos parámetros debido a la compleja interacción entre la tecnología del proceso y la morfología filamentosa de los hongos (Posch et al., 2013).

Por lo anterior, se han diseñado diversas configuraciones de biorreactores para la producción de hongos filamentosos como Trichoderma, tales como el tanque agitado (Ahamed y Vermette, 2009), el air-lift de tubo concéntrico (Ahamed y Vermette, 2010), etc., con la finalidad de optimizar estos procesos. El uso de biorreactores con agitación neumática ha resultado más eficiente ya que la agitación mecánica provoca un mayor esfuerzo de corte, y por lo tanto, la ruptura de los filamentos, provocando así la disminución de la actividad enzimática (Ahamed y Vermette, 2010). Es por ello que se han enfocado las investigaciones en el campo de la biotecnología de hongos utilizando biorreactores air-lift que favorecen la transferencia de masa, disminuyen el esfuerzo de corte, y minimizan el consumo de energía (Fontana et al., 2009; Mahmood et al., 2015; de Jesús et al., 2017), además, favorecen la formación de pellets que permiten periodos prolongados de producción de celulasas con biomasa fúngica retenida (Callow et al., 2016). En la Fig. 2 se presenta un esquema general de las etapas que constituyen un bioproceso para la obtención de metabolitos de interés biotecnológico a partir de hongos filamentosos.

En la última década se ha implementado el término biorrefinería, definido como el procesamiento sustentable de biomasa en un espectro de productos comerciables y energía, en el cual los hongos tienen un papel muy importante por su capacidad de utilizar biomasa lignocelulósica obtenida de los residuos de la agricultura y la actividad forestal (Castrillo et al., 2015; Fang y Xia, 2015) para la producción de bioenergía (Castrillo et al., 2015; Ajijolakewu et al., 2017; Canabarro et al., 2017).

Entre los residuos vegetales lignocelulósicos más utilizados para generar energía, se tienen al bagazo de caña de azúcar y residuos de soja, los cuales pueden ser degradados por diversos hongos filamentosos como Trichoderma, Aspergillus, Phlebia y Pleurotus, capaces de generar azúcares simples por efecto de rompimientos enzimáticos del residuo. Posteriormente, estos azúcares simples son fermentados por $S$. cerevisiae para producir etanol (Ahamed y Vermette, 2009; Chang y Webb, 2017). En México, los residuos ricos en celulosa que pueden ser utilizados para producir etanol son los residuos derivados de desechos agroindustriales, cultivos maderables, o residuos forestales, etc. (Aburto et al., 2008).

Existen diversas empresas dedicadas a la producción de etanol en Brasil y Estados Unidos; en Canadá una empresa produce este biocombustible a partir de residuos lignocelulósicos de trigo, avena y paja de cebada, con un rendimiento de 0,52 millones de galones de etanol por año (Dashtban et al., 2009). México cuenta con destilerías en los ingenios azucareros que permiten producir etanol destinado para bebidas y usos industriales; sin embargo, ante la crisis energética se ha planteado la producción de etanol anhidro utilizado como materia prima 
Aislamiento del hongo

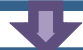

Propagación del microorganismo en medio definido a diferentes escalas

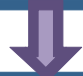

Selección de condiciones de cultivo: $\mathrm{pH}$, temperatura, humedad, aireación

Selección del sustrato(residuos lignocelulósicos)

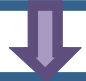

Pretratamiento físico o químico

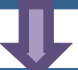

Hidrolisis o sacarificación

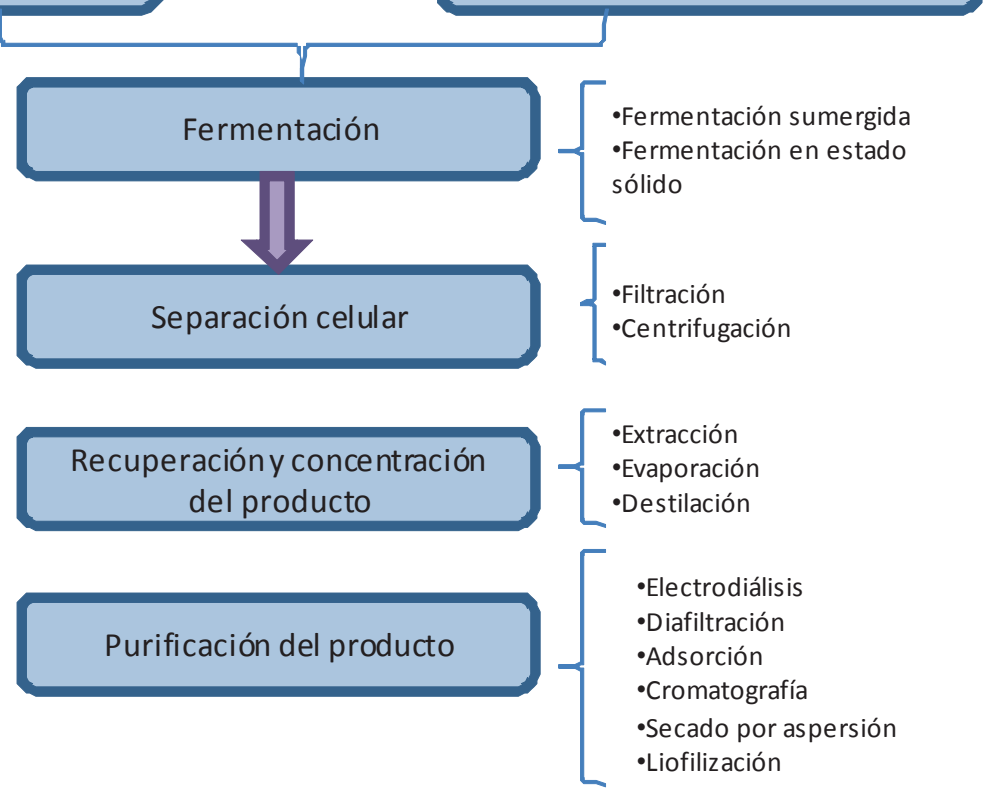

Fig. 2. Etapas generales de un bioproceso para la producción de metabolitos a partir de hongos. Fig. 2. General stages of a bioprocess for the production of fungal-derived metabolites.

para oxigenar las gasolinas y como sustituto de las mismas (Becerra-Pérez, 2009). Recientemente, se ha dado apoyo para la creación de una planta productora de bioetanol en Veracruz, con una capacidad instalada de 30 millones de litros al año (SAGARPA, 2017).

Otro de los biocombustibles que pueden ayudar a la crisis energética generada por el agotamiento de los combustibles fósiles es el biodiésel. En este aspecto, T. reesei mediante fermentación sumergida y utilizando ácido oleico como inductor, demostró un alto rendimiento de lípidos por peso seco de biomasa igual a $32,4 \%$, de los cuales el $49,7 \%$ correspondieron a ácidos grasos saturados susceptibles a un proceso de trans-esterificación para la generación de biodiésel; además, cabe destacar que se utilizan operaciones unitarias simples como la filtración y la ultrasonicación para obtener los lípidos totales del hongo (Bharathiraja et al., 2017).

Como se puede apreciar en esta revisión, con el paso de los años se han identificado nuevos compuestos producidos por diversas especies de Trichoderma, cuyo interés en la agricultura y en la biotecnología ha incrementado; por ello es que el aislamiento y la identificación de nuevas especies de este género fúngico, y la caracterización y efectividad biológica de sus metabolitos resultan de interés para diversas industrias. No obstante, es necesario fortalecer la investigación básica para identificar y evaluar metabolitos secundarios derivados de las especies de Trichoderma, y a la vez, enfocar ensayos tanto in vitro como in vivo para evaluar su efectividad biológica en plantas bajo diferentes condiciones. Lo anterior, permitirá validarlos al momento de aplicarlos en ensayos en condiciones de campo.

\section{AGRADECIMIENTOS}

Al Consejo Nacional de Ciencia y Tecnología (CONACyT) y al Colegio de Postgraduados por 
el apoyo asignado para la ejecución de la estancia posdoctoral de DJ H-M.

\section{LITERATURA CITADA}

Abdel-Mohsen, S.I., H.I. Abo-Elmagd, and M.M. Housseiny. 2016. A safe potential juice clarifying pectinase from Trichoderma viride EF-8 utilizing Egyptian onion skins. Journal of Genetic Engineering and Biotechnology 14:153-159.

Aburto, J., T. Martínez, y F. Murrieta. 2008. Evaluación técnico-económica de la producción de bioetanol a partir de residuos lignocelulósicos. Tecnología, Ciencia, Educación 23(1):23-30.

Ahamed, A., and P. Vermette. 2008a. Culturebased strategies to enhance cellulase enzyme production from Trichoderma reesei RUT-C30 in bioreactor culture conditions. Biochemical Engineering Journal 40:399-407.

Ahamed, A., and P. Vermette. 2008b. Enhanced enzyme production from mixed cultures of Trichoderma reesei RUT-C30 and Aspergillus niger LMA grown as fed batch in a stirred tank bioreactor. Biochemical Engineering Journal 42:41-46.

Ahamed, A., and P. Vermette. 2009. Effect of culture medium composition on Trichoderma reesei's morphology and cellulase production. Bioresource Technology 100:5979-5987.

Ahamed, A., and P. Vermette. 2010. Effect of mechanical agitation on the production of cellulases by Trichoderma reesei RUT-C30 in a draft-tube airlift bioreactor. Biochemical Engineering Journal 49:379-387.

Ajijolakewu, K.A., C.P. Leh, C.K. Lee, and W.A.W. Nadiah. 2017. Characterization of novel Trichoderma hemicellulase and its use to enhance downstream processing of lignocellulosic biomass to simple fermentable sugars. Biocatalysis and Agricultural Biotechnology 11:166-17.

Al-Hazmi, A.S., and M.T. Javeed. 2016. Effects of different inoculum densities of Trichoderma harzianum and Trichoderma viride against Meloidogyne javanica on tomato. Saudi Journal of Biological Sciences 23:288-292.

Argumedo-Delira, R., A. Alarcón, R. FerreraCerrato, y J.J. Peña-Cabriales. 2009. El género fúngico Trichoderma y su relación con contaminantes orgánicos e inorgánicos. Revista Internacional de Contaminación Ambiental 25(4):257-269.
Asad, S.A., A. Tabassum, A. Hameed, A. Afzal, S.A. Khan, R. Ahmed, et al. 2015. Determination of lytic enzyme activities of indigenous Trichoderma isolates from Pakistan. Brazilian Journal of Microbiology 46(4):1053-1064.

Ashok, A., K. Doriya, D.R.M. Rao, and D.S. Kumar. 2017. Design of solid state bioreactor for industrial applications: an overview to conventional bioreactors. Biocatalysis and Agricultural Biotechnology 9:11-18.

Azin, M., R. Moravej, and D. Zareh. 2007. Self-directing optimization of parameters for extracellular chitinase production by Trichoderma harzianum in batch mode. Process Biochemistry 34(6-7):563-566.

Bach, C., J. Yang, H. Larsson, S.M. Stocks, K.V. Gernaey, M.O. Albaek, et al. 2017. Evaluation of mixing and mass transfer in a stirred pilot scale bioreactor utilizing CFD. Chemical Engineering Science 171:19-26.

Bagewadi, Z.K., S.I. Mulla, and H.Z. Ninnekar. 2017. Purification and immobilization of laccase from Trichoderma harzianum strain HZN10 and its application in dye decolorization. Journal of Genetic Engineering and Biotechnology 15:139-150.

Barragán-Ocaña, A., and M. del C. del-ValleRivera. 2016. Rural development and environmental protection through the use of biofertilizers in agriculture: An alternative for underdeveloped countries?. Technology in Society 46:90-99.

Barreto, S.M., Ó.J.S. Toro, y L.F.G. Mosquera. 2011. Representación matemática de la producción de enzimas lignocelulolíticas por fermentación en estado sólido empleando Coriolus versicolor. Vector 6:63-70.

Becerra-Pérez, L.A. 2009. La industria del etanol en México. ECONOMÍA UNAM 6(16):82-98.

Bharathiraja, B., V. Sowmya, S. Sridharan, D. Yuvaraj, J. Jayamuthunagai, and R. Praveenkumar. 2017. Biodiesel production from microbial oil derived from wood isolate Trichoderma reesei. Bioresource Technology 239:538-541.

Callow, N.V., C.S. Ray, M.A. Kelbly, and L.K. Ju. 2016. Nutrient control for stationary phase cellulase production in Trichoderma reesei Rut C-30. Enzyme and Microbial Technology 82:8-14.

Canabarro, N.I., C. Alessio, E.L. Foletto, R.C. Kuhn, W.L. Priamo, and M.A. Mazutti. 2017. Ethanol production by solid-state saccharification and fermentation in a packed-bed bioreactor. Renewable Energy 102:9-14. 
Castrillo, M., G. Bich, G. Kramer, J. Velazquez, M. Rodriguez, P. Zapata, et al. 2015. Evaluation of synthetic and semi- synthetic culture media for endo- $1,4-\beta$ - glucanases secretion by Trichoderma koningiopsis. Procedia Materials Science 8:786-792.

Chang, C.W., and C. Webb. 2017. Production of a generic microbial feedstock for lignocellulose biorefineries through sequential bioprocessing. Bioresource Technology 227:35-43.

Charoenrak, P., and C. Chamswarng. 2016. Efficacies of wettable pellet and fresh culture of Trichoderma asperellum biocontrol products in growth promoting and reducing dirty panicles of rice. Agriculture and Natural Resources 50:243-249.

Chen, J.L., S.Z. Sun, C.P. Miao, K. Wu, Y.W. Chen, L.H. Xu, et al. 2016. Endophytic Trichoderma gamsii YIM PH30019: a promising biocontrol agent with hyperosmolar, mycoparasitism, and antagonistic activities of induced volatile organic compounds on root-rot pathogenic fungi of Panax notoginseng. Journal of Ginseng Research 40:315-324.

Cumagun, C.J.R. 2014. Advances in formulation of Trichoderma for biocontrol. p. 527-532. En V.K. Gupta, M. Schmoll, A. HerreraEstrella, R.S. Upadhyay, I. Druzhinina, y M. Tuohy (eds.) Biotechnology and Biology of Trichoderma. Amsterdam, Netherlands.

Dashtban, M., H. Schraft, and W. Qin. 2009. Fungal bioconversion of lignocellulosic residues; opportunities \& perspectives. International Journal of Biological Sciences 5(6):578-595.

de Aguiar, R.A., M.G. da Cunha, and M.L. Junior. 2014. Management of white mold in processing tomatoes by Trichoderma spp. and chemical fungicides applied by drip irrigation. Biological Control 74:1-5.

de Jesús, S.S., N.J. Moreira, and F.R. Maciel. 2017. Hydrodynamics and mass transfer in bubble column, conventional airlift, stirred airlift and stirred tank bioreactors, using viscous fluid: a comparative study. Biochemical Engineering Journal 118:70-81.

Delabona, P. da S., C.S. Farinas, D.J. da S. Lima, and J.G. da C. Pradella. 2013. Experimental mixture design as a tool to enhance glycosyl hydrolases production by a new Trichoderma harzianum P49P11 strain cultivated under controlled bioreactor submerged fermentation. Bioresource Technology 132:401-405.
Diánez, M.F., M. Santos, F. Carretero, and F. Marín. 2016. Trichoderma saturnisporum, a new biological control agent. Journal of the Science of Food and Agriculture 96(6):19341944.

Dieleman, H. 2016. Urban agriculture in Mexico City; Balancing between ecological, economic, social and symbolic value. Journal of Cleaner Production 163:S156-S163.

Diorio, L.A., F. Forchiassin, V.L. Papinutti, y D.V. Sueldo. 2003. Actividad enzimática y degradación de diferentes tipos de residuos orgánicos por Saccobolus saccoboloides (Fungi, Ascomycotina). Revista Iberoamericana de Micología 20:11-15.

Djonovic, S., W.A. Vargas, M.V. Kolomiets, M. Horndeski, A. Wiest, and C.M. Kenerley. 2007. A proteinaceous elicitor Sm1 from the beneficial fungus Trichoderma virens is required for induced systemic resistance in maize. Plant Physiology 145:875-889.

Doran, P. M. 1998.Principios de ingeniería de los bioprocesos. Acribia, Zaragoza, España.

Fadel, H.H.M., M.G. Mahmoud, M.M.S. Asker, and S.N. Lotfy. 2015. Characterization and evaluation of coconut aroma produced by Trichoderma viride EMCC-107 in solid state fermentation on sugarcane bagasse. Electronic Journal of Biotechnology 18:5-9.

Fang, H., and L. Xia. 2015. Cellulase production by recombinant Trichoderma reesei and its application in enzymatic hydrolysis of agricultural residues. Fuel 143:211-216.

Fontana, R.C., T.A. Polidoro, and M.M. da Silveira. 2009. Comparison of stirred tank and airlift bioreactors in the production of polygalacturonases by Aspergillus oryzae. Bioresource Technology 100:4493-4498.

FAO. 2014. Growing Greener Cities in Latin America and the Caribbean. Food and Agricultural Organization of the United Nations (FAO), Santiago, Chile. Disponible en http://www.fao.org/3/a-i3696e.pdf (Consulta 15 enero 2018)

Fuglie, K. 2016. The growing role of the private sector in agricultural research and development world-wide. Global Food Security 10:29-38.

García de León, S., y T. Mier. 2010. Visión general de la producción y aplicación de bioplaguicidas en México. Sociedades rurales, producción y medio ambiente 10(20):37-63.

García-Espejo, C.N., M.M. Mamani-Mamani, G.A. Chávez-Lizárraga, y M.T. Álvarez-Aliaga. 2016. Evaluación de la actividad enzimática del Trichoderma inhamatum (BOL-12 QD) como posible biocontrolador. Journal of the Selva Andina Research Society 7(1):20-32. 
Guoweia, S., H. Man, W. Shikai, and C. He. 2011. Effect of some factors on production of cellulase by Trichoderma reesei HY07. Procedia Environmental Sciences 8:357-361.

Hohmann, P., E.E. Jones, R.A. Hill, and A. Stewart. 2011. Understanding Trichoderma in the root system of Pinus radiata: associations between rhizosphere colonisation and growth promotion for commercially seedlings. Fungal Biology UK 115:759-767.

Ibraheem, O., and B.K. Ndimba. 2013. Molecular adaptation mechanisms employed by ethanologenic bacteria in response to lignocellulose derived inhibitory compounds. International Journal of Biological Sciences 9(6):598-612.

Idris, A.S.O., A. Pandey, S. Rao, and R.K. Sukumaran. 2017. Cellulase production through solid state tray fermentation, and its use for bioethanol from sorghum stover. Bioresource Technology 242:265-271.

Jaklitsch, W.M., and H. Voglmayr. 2015. Biodiversity of Trichoderma (Hypocreaceae) in Southern Europe and Macaronesia. Studies in Mycology 80:1-87.

Kent, J.A., T.V. Bommaraju, and S.D. Barnicki. 2017. Handbook of Industrial Chemistry and Biotechnology. Springer, New York, USA.

Komarek, A.M., S. Drogue, R. Chenoune, J. Hawkins, S. Msangi, H. Belhouchette, and G. Flichman. 2017. Agricultural household effects of fertilizer price changes for small holder farmers in central Malawi. Agricultural Systems 154:168-178.

Kumar, S., M. Thaku, and A. Rani. 2014. Trichoderma: Mass production, formulation, quality control, delivery and its scope in commercialization in India for the management of plant diseases. African Journal of Agricultural Research 9(53):38383852.

la Grange, D.C., R. den Haan, and W.H. van Zyl. 2010. Engineering cellulolytic ability into bioprocessing organisms. Applied Microbiology and Biotechnology 87:11951208.

Mahmood, K.H.A.M., S.J. Wilkinson, and W.B Zimmerman. 2015. Airlift bioreactor for biological applications with microbubble mediated transport processes. Chemical Engineering Science 137:243-253.

Marcello, C.M., A.S. Steindorff, S.P. Silva, R.N. Silva, L.A.M. Bataus, and C.J. Ulhoa. 2010. Expression analysis of the exo- $\beta-1,3-$ glucanase from the mycoparasitic fungus Trichoderma asperellum. Microbiological Research 165:75-81.
Marques, S., C.T. Matos, F.M. Gírio, J.C. Roseiro, and J.A.L. Santos. 2017. Lactic acid production from recycled paper sludge: Process intensification by running fedbatch into a membrane-recycle bioreactor. Biochemical Engineering Journal 120:63-72.

Martínez, B., D. Infante, y Y. Reyes. 2013. Trichoderma spp. y su función en el control de plagas en los cultivos. Revista de Protección Vegetal 28(1):1-11.

Morgan, C.A., N. Herman, P.A. White, and G. Vesey. 2006. Preservation of microorganisms by drying:a review. Journal of Microbiological Methods 66:183-193.

Navaneetha, T., R.D. Prasad, and R.L. Venkateswara. 2015. Liquid formulation of Trichoderma species for management of gray mold in castor (Ricinus communis L.) and Alternaria leaf blight in sunflower (Helianthus annuus L.). Journal of Biofertilizers and Biopesticides 6(1):149.

Posch, A.E., C. Herwig, and O. Spadiut. 2013. Science-based bioprocess design for filamentous fungi. Trends in Biotechnology 31(1): 37-44.

Ramos, E.Y.A., R.I.Z. Navarro, L.E.O. Zumaqué, y J.L.B. Violeth. 2008. Evaluación de sustratos y procesos de fermentación sólida para la producción de esporas de Trichoderma sp. Revista Colombiana de Biotecnología $X(2): 23-34$.

Romero-Cortes, T., P.A. López-Pérez, M. RamírezLepe, y J.A. Cuervo-Parra. 2016. Modelado cinético del micoparasitismo por Trichoderma harzianum contra Cladosporium cladosporioides aislado de frutos de cacao (Theobroma cacao). Chilean Journal of Agricultural and Animal Science, ex Agro-Ciencia 31(3):32-45.

Ruiz, H.A., R.M. Rodríguez-Jasso, R. Rodríguez, J.C. Contreras-Esquivel, and C.N. Aguilar. 2012. Pectinase production from lemon peel pomace as support and carbon source in solid-state fermentation column-tray bioreactor. Biochemical Engineering Journal 65:90-99.

Sandle, T. 2014. Trichoderma. p. 644-646. En C.A. Batt y M.-L. Tortorello (eds.) Encyclopedia of Food Microbiology, London, UK.

SAGARPA. 2017. Impulsa SAGARPA producción de biocombustibles en México. Comunicado de Prensa Secretaría de Agricultura, Ganadería, Desarrollo Rural, Pesca y Alimentación (SAGARPA). CDMX, México. Disponible en https://www.gob.mx/sagarpa/ es/prensa/impulsa-sagarpa-produccionde-biocombustibles-en-mexico?idiom $=e s$ (Consulta 11 febrero 2018). 
Sharma,V., R. Salwan, and P.N. Sharma. 2017. The comparative mechanistic aspects of Trichoderma and probiotics: scope for future research. Physiological and Molecular Plant Pathology 100:84-96.

Soccol, C.R., E.S.F. da Costa, L.A.J. Letti, S.G. Karp, A.L. Woiciechowski, and L.P. de S. Vandenberghe. 2017. Recent developments and innovations in solid state fermentation. Biotechnology Research and Innovation 1(1):52-71.

Subramaniyam, R., and R. Vimala. 2012. Solid state and submerged fermentation for the production of bioactive substances: a comparative study. International Journal of Security and Networks 3(3):480-486.

Tejeda, A. 2011. Bioseparaciones. Pearson, México.

Torres-De la Cruz, M., C.F. Ortiz-García, C. Bautista-Muñoz, J.A. Ramírez-Pool, N. Ávalos-Contreras, S. Cappello-García, et al. 2015. Diversidad de Trichoderma en el agroecosistema cacao del estado de Tabasco, México. Revista Mexicana de Biodiversidad 86:947-961.

Vargas-Hoyos, H.A, y E. Gilchrist-Ramelli. 2015. Producción de enzimas hidrolíticas y actividad antagónica de Trichoderma asperellum sobre dos cepas de Fusarium aisladas de cultivos de tomate (Solanum lycopersicum). Revista Mexicana de Micología 42:9-16.
Vinale, F., K. Sivasithamparam, E.L. Ghisalberti, R. Marra, M.J. Barbetti, H. Li, et al. 2008. A novel role for Trichoderma secondary metabolites in the interactions with plants. Physiological and Molecular Plant Pathology 72:80-86

Vuppala, G., R. Krishna, and K. Murthy. 2015. Industrial Fermentation. Research and Reviews: Journal of Microbiology and Biotechnology (RRJET) 4(1):1-7.

Woo, S.L., M. Ruocco, F. Vinale, M. Nigro, R. Marra, N. Lombardi, et al. 2014. Trichodermabased products and their widespread use in agriculture. The Open Mycology Journal 8(Suppl-1, M4):71-126.

Zeilinger, S., S. Gruber, R. Bansal, and P.K. Mukherjee. 2016. Secondary metabolism in - chemistry meets genomics. Fungal Biology Reviews 30(2):74-90. 